\title{
CONFORMATIONAL EQUILIBRIA INVOLVING SUBSTITUENTS LACKING CONICAL SYMMETRY
}

\author{
M. J. T. ROBINSON \\ Dyson Perrins Laboratory, University of Oxford, Oxford
}

\begin{abstract}
The methods available for studying conformational equilibria are discussed with emphasis on the problems posed by substituents of low symmetry. The topics considered include inversion of the $\mathrm{N}$ atom in piperidines, solvent and hydrogen isotope effects in 1,3-dioxanes, rotational isomerism of alkyl substituents, and equilibria in molecules with two polar groups.
\end{abstract}

I chose the subject of this paper because it would involve discussing features of conformational analysis which are important for cyclic compounds but which are sometimes neglected or misunderstood and which illustrate present limitations in our understanding and techniques. In the first part I will discuss some methods of determining conformational equilibria in six-membered ring compounds and some of the results for substituents lacking conical symmetry†. In the second part I will discuss the problem of dipole-dipole interactions in compounds with two or more polar groups, whether as substituents or elements of a ring, and some misunderstandings about such interactions. This will include conically symmetrical substituents frequently but only incidentally to my main topic.

As a prelude I must explain what I mean by 'conformation' and therefore by conformational equilibrium. 'Conformations' have often been described in some such way as, 'the non-identical arrangements of the atoms obtainable by rotation about one or more single bonds ${ }^{1 \text { a }}$. Fortunately the word is seldom used consistently in this sense, which, for example, excludes the chair form from being counted as one of the conformations of cyclohexane! This sense also makes the term 'conformational equilibrium' meaningless because a conformation so defined is a hypothetical structure which may be assigned a potential energy but cannot have thermodynamic functions, and cannot be in equilibrium with another conformation. Irrespective of the definition given, 'conformation' is commonly used in the sense of conformer or conformational isomer, or in some related manner which is consistent with the customary looseness of English usage.

Conformational isomerism may be regarded as a subdivision of stereoisomerism in the same sense that the latter is a subdivision of isomerism.

$\dagger$ Conically symmetrical substituents are those with $\mathrm{C}_{\infty o v}$ symmetry, e.g. $-\mathrm{Cl} .-\mathrm{CN}$, and must be distinguished from axially symmetrical substituents such as phenyl and methyl, which are sometimes wrongly referred to as conically or even spherically symmetrical. 


\section{J. T. ROBINSON}

Conformations are stereoisomeric structures in mobile equilibrium and are interconvertible by stretching and bending of bonds and by rotation about formal single bonds. When the free energy of activation becomes high enough for separation to be possible under ordinary conditions, e.g. in optically resolvable biphenyls ${ }^{2}$, such structures are regarded as ordinary stereoisomers. When the energy barriers are too low for separation to be feasible near room temperature but do allow the detection of distinguishable molecules the latter are called conformations. When the energy barrier is very low but there are still two or more sets of vibration levels corresponding to 'wells' in a potential energy surface, e.g. formamide ${ }^{3}$, which is non-planar in the lowest vibration state but planar, with a highly anharmonic out-of-plane vibration, in the first excited vibration state, the distinction between the various conformations may often be ignored for convenience. When, however, the potential energy 'wells' are so shallow that even the lowest vibration state has a higher energy than the barrier separating them, e.g. oxetane ${ }^{4}$, then there is only one conformation, as is also the case, of course, with compounds with but a single potential energy minimum $\dagger$.

In six-membered compounds the term conformational equilibrium usually applies to either the equilibrium between the chair and boat forms, the boat being loosely regarded as a single conformation, or to the equilibrium between two chair conformations with one or more substituents axial or equatorial. When the substituent is not conically symmetrical, however, there are further conformational equilibria possible involving rotation of the substituent about the bond joining it to the ring, and it is then sometimes convenient to refer to axial or equatorial 'sets' of conformations whenever axial or equatorial conformation alone would be ambiguous ${ }^{6,7}$.

Our interest has been centred around six-membered ring compounds for several reasons. They are second in importance only to acyclic compounds but are often conformationally much simpler than the latter. This is because the two chair conformations of rings containing not more than one trigonal atom are usually considerably more stable than the boat conformations, have nearly normal bond and torsion angles, so that results for acyclic compounds are directly relevant, have limited possibilities for substituents, and offer the widest range of methods for studying conformational equilibria.

The methods used for studying conformational equilibria often use one or more relationships of the following type:

$$
X=\sum_{i}^{n} N_{i} X_{i}
$$

where $X$ is the value of some property of the compound, $N_{i}$ is the mole fraction of the $i$ th conformation and $X_{i}$ is the corresponding value of the property. The methods most commonly used for studying conformational equilibria may be divided into five groups :

(a) Direct methods primarily suited to determining structure (e.g. electron diffraction and microwave spectroscopy) and as yet offering rather low accuracy for estimating concentrations, and limited to simple structures. These will not be referred to again.

(b) Fast chemical reactions.

† A similar description of the use of the word 'conformation' has been given by Miller ${ }^{5}$. 
(c) Direct spectroscopic methods in which particular absorption bands can be assigned to specific conformations.

(d) The use of stereochemical equilibria as models for conformational equilibria.

(e) Chemical and physical methods in which the value of some observable property of a compound is some weighted average of the values of the individual conformations.

If a different stereoisomer results from a quantitative stereospecific reaction of each of the conformations in a conformational equilibrium, i.e. if the chemical reaction is much faster than the conformational change so that the Curtin-Hammett Principle does not apply, then relation (1) reduces to a very simple form:

$$
X(i)=N_{i} \quad(i=1 \text { to } n)
$$

where $X(i)$ is the fraction of the total product that is the stereoisomer formed from the $i$ th conformation. Unfortunately, few stereospecific reactions are faster than conformational changes. One such reaction is the protonation of strong bases by strong acids. Booth ${ }^{8}$ has used the reaction between liquid cis-3,5-dimethylpiperidine (I) and deuterotrifluoroacetic acid to determine the conformational equilibrium in the former (Figure 1). He assumed that because there is no isotopic scrambling between solute and solvent there is no scrambling between solute molecules, i.e. only the ions $\mathrm{II}(\mathrm{a}-\mathrm{H})$ and $\mathrm{II}(\mathrm{e}-\mathrm{H})$ are formed stereospecifically from Ia and le. This assumption has been questioned $^{9}$ and shown to be wrong for other amines ${ }^{10}$. When liquid dimethylamine, for example, is mixed with deuterotrifluoroacetic acid a nearly statistical mixture of the ions $\mathrm{Me}_{2} \mathrm{NH}_{2}, \mathrm{Me}_{2} \stackrel{+}{\mathrm{N}} \mathrm{HD}$, and $\mathrm{Me}_{2} \mathrm{ND}_{2}$, easily distinguishable from their nuclear magnetic resonance spectra, results ${ }^{10}$. Although protonation is almost certainly much faster than inversion at a nitrogen atom, the mixing of liquids is a slow process affording ample opportunity for exchange between amine and ion, and indeed when liquid tertiary amines are mixed with trifluoroacetic acid the compositions of the solutions approximate to the equilibrium composition for the ions $s^{9,10}$. A variant of Booth's method, however, does appear to be valid. When dimethylamine vapour is allowed to diffuse slowly to the surface of concentrated deuteriosulphuric acid isotopic scrambling for dimethylamine is almost eliminated and the sole reaction appears to be fast protonation of separate amine molecules as they reach the surface of the $\operatorname{acid}^{10}$. When applied to $N$-methylpiperidines this method indicates a much greater preponderance of conformations with the $N$-methyl group equatorial than has been found in the most recent dipole moment study ${ }^{11}$. This conflict of evidence is intriguing because the dipole moment studies were made with care, while the protonation is elegant and direct.

Two aspects of the third group of methods are to be considered briefly. A problem with most spectroscopic methods of this type is that when two well separated absorption bands can be found the values of $X_{i}$ in relation (1), e.g. extinction coefficients or integrated intensities for an individual conformation in infra-red spectra, cannot always be determined. Nevertheless this method can be very effective but its application to the orientations of the hydroxyl group in a cyclohexanol illustrates an important limitation. The 
<smiles>CC1CC[NH+]([O-])CC(C)C1</smiles>

Io<smiles>[M]C(C)C(C)C[N+]([2H])([2H])CCCC</smiles>

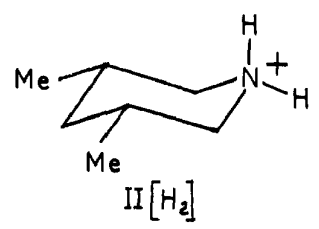

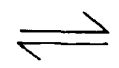<smiles>CC1CCNCCC(C)C1</smiles>

Ie

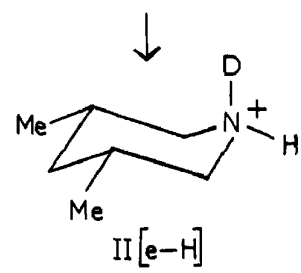

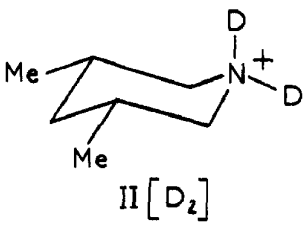

Figure 1. Kinetically controlled salt formation from cis-3,5-dimethylpiperidine and a strong deuterio-acid $^{8,10}$. The presently available N.M.R. method of analysis does not readily allow a distinction between $\mathrm{II}(\mathrm{a}-\mathrm{H})$ and $\mathrm{II}\left(\mathrm{H}_{2}\right)$, and between $\mathrm{II}(\mathrm{e}-\mathrm{H})$ and $\mathrm{II}\left(\mathrm{D}_{2}\right)$. All four ions are expected to be formed ${ }^{10}$ when a liquid amine is mixed with a liquid acid, e.g. $\mathrm{CF}_{3} \mathrm{CO}_{2} \mathrm{D}^{8}$, but only $\mathrm{II}(\mathrm{a}-\mathrm{H})$, from Ia, and II (e-H), from Ie, when the amine vapour diffuses slowly to the surface of an involatile acid, e.g. $\mathrm{D}_{2} \mathrm{SO}_{4}{ }^{10}$.

symmetry of the $\mathrm{OH}$ stretching band when the $\mathrm{OH}$ is axial has been taken as evidence that only one type of conformation is present, that with the hydroxyl group pointing out of the ring, but it has been argued that both types of conformation are present in substantial amount but have nearly coincident stretching frequencies ${ }^{12,13}$. The problem can only be solved by using properties, e.g. vicinal coupling constants for the fragment $\mathrm{H}-\mathrm{C}-\mathrm{O}-\mathrm{H}^{13} \dagger$ or dipole moments of 4-arylcyclohexanols, which differ in a predictable way for the two types of conformation.

Nuclear magnetic resonance spectra are free from the difficulty of determining extinction coefficients or similar parameters, and as there appear to be no unavoidable errors ${ }^{14}$ this technique can provide reliable data to test other methods for determining conformational equilibria. Unfortunately, integration of nuclear magnetic resonance spectra can only be used in this way at temperatures at which the conformational equilibrium is slow enough for separate spectra to be observed. This is a severe limitation and it is commonly possible to measure a free energy difference effectively at only one temperature for chair-chair equilibria ${ }^{14}$. In addition, it is not possible to

$\dagger$ Because it will be necessary to use very dilute solutions to eliminate intermolecular hydrogen bonding and because it is difficult to eliminate chemical exchange of $\mathrm{OH}$-protons in the absence of hydrogen bonding such measurements will be very difficult ${ }^{13}$. In contrast, dipole moments are best measured in very dilute solutions in non-polar solvents and a study of 4-p-nitrophenylcyclohexanol has been begun by $\mathrm{Mr}$ J. D. Schofield at Oxford, the dipole moments to be measured in collaboration with Dr L. E. Sutton in the Physical Chemistry Laboratory. 


\section{SUBSTITUENTS LACKING CONICAL SYMMETRY}

slow down the very mobile equilibria within axial or equatorial sets of conformations, so that individual conformations cannot be observed when a substituent has low symmetry. Finally, the present precision in integrating nuclear magnetic resonance spectra makes it difficult to measure accurately equilibrium constants greater than about 20 , which corresponds to a free energy difference of $\sim \pm 1 \mathrm{kcal} / \mathrm{mole}$ at $-100^{\circ}$, at which temperature many, but by no means all, chair-chair equilibria are slow. We have studied 5methyl-1,3-dioxane ${ }^{15}$, in which the axial and equatorial methyl groups give fairly sharp, well-separated resonances at $\sim-90^{\circ 15}$. The free energy differences for three different solvents (Table 1 ) are markedly higher than previous estimates based on equilibration of several pairs of stereoisomers ${ }^{17-19}$, admittedly in different solvents, but agree with results derived from vicinal coupling constants ${ }^{15}$ and chemical shifts ${ }^{16}$. This is a rather serious failure of the use of stereochemical equilibra as models for conformational equilibria. The small differences between the three solvents $\mathrm{CCl}_{3} \mathrm{~F},\left(\mathrm{CD}_{3}\right)_{2} \mathrm{CO}$, and $\mathrm{CD}_{3} \mathrm{OD}$ are barely significant but are the opposite of what might have been expected, because the 5-axial methyl group should hinder solvation of the oxygen atoms. This result may arise from dipoledipole repulsions, since it has been shown that methyl is weakly electron attracting relative to hydrogen when attached to $s p^{3}$ carbon that does not carry electronegative substituents.

Table 1. Estimates of free energy differences $\Delta G_{e \rightarrow a}$ for 1,3-dioxane

\begin{tabular}{|c|c|c|c|c|}
\hline Method & Solvent & $\underset{{ }^{\circ} \mathrm{Kemp}}{\mathrm{T}}$ & $\underset{\mathrm{kcal} / \mathrm{mole}}{\Delta G_{e \rightarrow a}}$ & Ref. \\
\hline \multicolumn{5}{|l|}{ Equilibrium (3) ${ }^{a}$} \\
\hline \multirow[t]{2}{*}{$\mathbf{R}=\mathbf{M e}$} & $\mathrm{Et}_{2} \mathrm{O}$ & 298 & 0.97 & 18 \\
\hline & none & - & 0.81 & 19 \\
\hline \multirow[t]{2}{*}{$\mathrm{R}=t-\mathrm{Bu}$} & $\mathrm{Et}_{2} \mathrm{O}$ & 298 & 0.80 & 18 \\
\hline & $\mathrm{CHCl}_{3}$ & 298 & 0.89 & 17 \\
\hline $\begin{array}{l}\mathbf{R}=p-\mathrm{NO}_{2} \mathrm{C}_{6} \mathrm{H}_{4} \\
\text { Equilibrium (4) }\end{array}$ & $\mathrm{CHCl}_{3}$ & 298 & 0.87 & 17 \\
\hline \multirow[t]{3}{*}{ N.M.R. integration ${ }^{b}$} & $\mathrm{CCl}_{3} \mathrm{~F}$ & 180 & 1.10 & 33 \\
\hline & $\left(\mathrm{CD}_{3}\right)_{2} \mathrm{CO}$ & 180 & 1.08 & 33 \\
\hline & $\mathrm{CD}_{3} \mathrm{OD}$ & 180 & 1.05 & 33 \\
\hline \multirow[t]{4}{*}{ Coupling constants ${ }^{\mathfrak{c}}$} & $\mathrm{CCl}_{3} \mathrm{~F}$ & 307 & 1.10 & 15 \\
\hline & $\left(\mathrm{CD}_{3}\right)_{2} \mathrm{CO}$ & 307 & 1.05 & 15 \\
\hline & $\left(\mathrm{CH}_{3}\right)_{4} \mathrm{Si}$ & 307 & 1.05 & 15 \\
\hline & $\mathrm{CD}_{3} \mathrm{OD}$ & 307 & 1.03 & 15 \\
\hline \multirow[t]{3}{*}{ Chemical shifts $^{d}$} & $\mathrm{CC}_{3} \mathrm{~F}$ & 307 & 1.1 & 16 \\
\hline & $\left(\mathrm{CD}_{3}\right)_{2} \mathrm{CO}$ & 307 & 1.1 & 16 \\
\hline & $\left(\mathrm{CH}_{3}\right)_{4} \mathrm{Si}$ & 307 & 1.05 & 16 \\
\hline
\end{tabular}

acid-catalysed; errors are generally about \pm 0.02 but the difference between the first two entries seems too large to be due to a solvent effect.

- Axial and equatorial 5-methyl resonances, the former being corrected for the overlapping ${ }^{13} \mathrm{C}$ satellites of the equatorial 5 -methyl group resonance; errors are about \pm 0.05 to \pm 0.08 for the individual values but rather smaller for differences between them.

- Vicinal coupling constants between trans protons at $C-4(6)$ and at $C-5$; errors are about +0.05 .

Difference between the chemical shifts of the geminal C-2 protons. Two sets of model compounds (Figure 5) were used to estimate by extrapolation the temperature dependence of the chemical shifts of the equatorial conformation, which were directly measurable at low temperatures, and to estimate the chemical shifts of the axial conformation. Although random errors are small the systematic errors are large (see text). 
The absorption due to the axial $t$-butyl group in 5-t-butyl-1,3-dioxane in $\mathrm{CCl}_{3} \mathrm{~F}$ at $-100^{\circ}$ is so small that even with a fairly sharp signal only very rough estimates of $\Delta G_{e \rightarrow a}(+2.0 \pm 0.4 \mathrm{kcal} / \mathrm{mole})$ can be made, but these seem to be a little higher than previous estimates ${ }^{17,18}$, in keeping with the observations for the methyl group.

The first three groups of methods provide no information about conformational equilibria within axial or equatorial sets of conformations of alkyl derivatives, but the fourth, the use of chemical equilibria as models for conformational equilibria, has some value. In this method one supposes that a stereochemical equilibrium, e.g. (3) is a valid model for the conformational
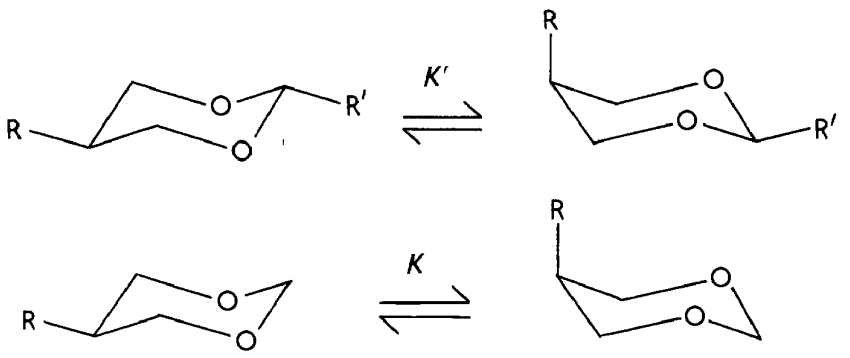

equilibrium (4), i.e. one assumes that the approximate equality in relationships (5)

$$
K^{\prime}=N_{2}^{\prime} / N_{1}^{\prime} \simeq N_{2} / N_{1}=K
$$

is not a significant source of error. When differences in thermodynamic functions for pairs of stereoisomers as in equation (3) may be derived either calorimetrically or from the equilibrium constants for an accurately reversible reaction studied over a wide range of temperature, not only free energy differences $\left(\Delta G_{e \rightarrow a}\right)$ but also enthalpies $\left(\Delta H_{e \rightarrow a}\right)$ and entropies $\left(\Delta S_{e \rightarrow a}\right)$ for the conformational equilibrium (4) may be derived. If it is assumed that the entropies of various individual conformations are equal, then $\Delta S_{e \rightarrow a}$ may be interpreted as the difference between the entropies of mixing for the axial and equatorial sets of conformations for substituents lacking conical symmetry†. Our own early attempts to do this were abortive because the high precision required was not achieved ${ }^{7}$, but more accurate measurements for polar substituents are still inconclusive ${ }^{20}$. Although the values of $\Delta S_{e \rightarrow a}$ are sometimes within the range expected for differences of entropies of mixing it is not yet possible for polar substituents in polar solvents or for selfassociated compounds to allow for solvation or association effects ${ }^{20}$. We have pointed out that differential effects of solvation on stereoisomers, and therefore on conformations so far as the use of models is valid, may be

$\mp$ The threefold axially symmetrical substituents such as methyl are almost always supposed by analogy with saturated acyclic compounds to prefer staggered orientations about the bond between the substituent and the ring, so that there are three indistinguishable conformations with the substituent either axial or equatorial and therefore the entropy of mixing is expected to be zero in all cases. Should the torsional frequencies of the methyl group be significantly different for the two conformations then there could be a significant entropy (and energy) difference between the two conformations which would be attributable to the substituent in a way that is different from any entropy effects associated with conically symmetrical substituents. 
investigated by gas-liquid chromatography (g.l.c. $)^{7,21}$. So far there has been no example of an equilibrium of type (3) set up in a liquid suitable for use as a stationary phase for determining the retention parameters of the stereoisomers, so that this unique advantage of the use of stereochemical equilibria as models for conformational equilibria has never been exploited.

Alkyl substituents are less likely to lead to complications due to solvation than are other groups but accurate data are not yet available for the series of alkylcyclohexanes with the alkyl groups methyl, ethyl and isopropyl. The very unsymmetrical environment of the alkyl groups in 2-alkylcyclohexanones, however, leads to widely differing values of $\Delta H_{e \rightarrow a}$ and $\Delta S_{e \rightarrow a}$ for methyl, ethyl and isopropyl groups and by making commonly accepted assumptions about the additivity of interactions a relatively complete analysis of the conformational equilibria was possible. The conformations of equatorial 2-isopropylcyclohexanone, for example, were deduced to have the relative stabilities IIIa $>b>c^{21}$, although this is not considered to be consistent with the circular dichroism of related optically active ketones ${ }^{22}$.

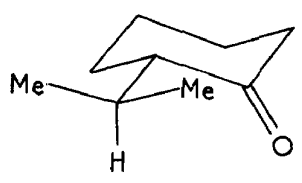

III a

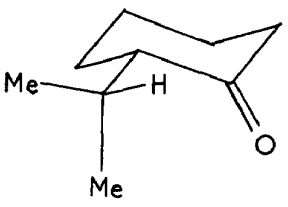

IIIS

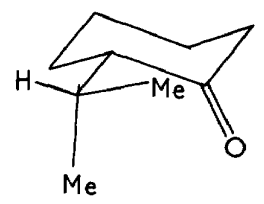

IIIC

The interactions derived from the conformational equilibria in 2-alkylcyclohexanones were then used to explain semi-quantitatively the difference between the equilibrium in propionaldehyde or isobutyraldehyde (in which the strongly preferred conformations have the carbonyl group eclipsed by a methyl group) and in diethylacetaldehyde (in which conformations resulting from rotation about the $\mathrm{C}-\mathrm{CO}$ bond are of nearly equal stability) ${ }^{21}$ and the signs and amplitudes of the Cotton effects of acyclic aldehydes and methyl ketones $^{23}$.

Enthalpy and entropy differences for the 5-alkyl-1,3-dioxanes have been measured with reasonable accuracy ${ }^{17}$. Two points are worth discussion. It has been noted previously but not explained ${ }^{18}$ that estimates of $\Delta G_{e \rightarrow a}$ are consistently a little lower for ethyl than for methyl. This is not due to an unexpected positive $\Delta S_{e \rightarrow a}{ }^{17}$, which, e.g., could result from a marked preference for one conformation when ethyl is equatorial. An effect which could raise the energy of an axial methyl group would be an increase in the torsional frequency and therefore zero point energy (probably at most 0.1 $\mathrm{kcal} / \mathrm{mole}$ ) resulting from the steric repulsions between the hydrogen atom of the axial methyl group pointing into the ring and the oxygen atoms. Because the other alkyl groups have a much larger moment of inertia than methyl for internal rotation this effect should be far smaller for them. The entropy differences for ethyl and isopropyl require that the axial conformations with a hydrogen atom or with a methyl group turned into the ring do not differ much 


\section{J. T. ROBINSON}

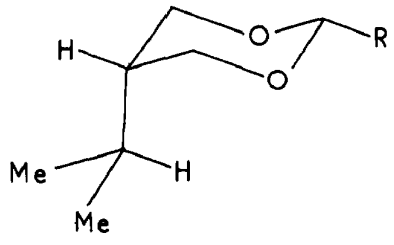

$$
\begin{aligned}
\mathrm{IVa}: \mathbf{R} & =\mathbf{H} \\
\mathrm{V}: \mathbf{R} & =\mathrm{Me} \\
J & =8.7 \mathrm{~Hz} ; N_{1}=0.52
\end{aligned}
$$<smiles>[R]C1OCC(C(C)C)CO1</smiles>

$$
\begin{aligned}
\text { IVe: } \mathrm{R} & =\mathrm{H} \\
\text { VI: } \mathrm{R} & =\mathrm{Me} \\
J & =7.8 \mathrm{~Hz} ; \quad N_{1}=0.42 \\
\Delta S_{\text {mix }} & =+0.11 \text { e.u. }
\end{aligned}
$$

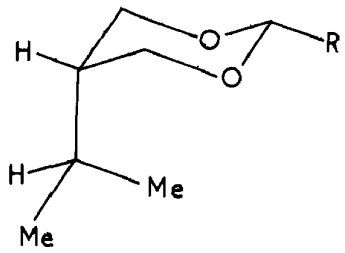

$$
N_{2}=N_{3}=0.24 \quad S_{\operatorname{mix}}=2.04 \text { e.u. }
$$<smiles>[R]C1OCCC(C(C)(C)C)CO1</smiles>

$$
\begin{gathered}
N_{2 .}=N_{3}=0.29 \quad S_{\text {mix }}=2.15 \text { e.u. } \\
\Delta S_{\text {obs }}=-0.1 \pm 0.2 \text { e.u. }
\end{gathered}
$$

Figure 2. Conformational equilibria within the axial and equatorial sets of conformations of 5-isopropyl-1,3-dioxane $(\mathbf{R}=\mathbf{H})$ and the cis- and trans-2-methyl derivatives $(\mathbf{R}=\mathbf{M e})$ used as models ${ }^{15}$. The derived value of the entropy of mixing is very insensitive to errors in the assumed values of the gauche and trans coupling constants for the 5- and 2'-protons. Conversely the observed entropy difference in this and many other instances has little value in the study of conformational equilibria within sets of conformations.

in stability (Figure 2), in agreement with the relatively low value of $\Delta G_{e \rightarrow a}$ for 5-t-butyl-1,3-dioxane ${ }^{17,18}$ and in contrast to ethyl- and isopropylcyclohexane with the alkyl group axial. The observed and calculated values of $\Delta S_{e \rightarrow a}$ agreed well for ethyl but less well for isopropyl and it seemed worthwhile to investigate more directly the equilibria within the axial and equatorial sets of conformations of 5-isopropyl-1,3-dioxane. This is essentially a problem in an acyclic system and can best be tackled by one of the fifth group of methods of studying conformational equilibria.

These methods depend on observing a weighted average of the properties of the individual conformations, and the most often used properties now are nuclear magnetic resonance chemical shifts and coupling constants (and related parameters such as band widths), at temperatures at which only time average values can be observed, and dipole moments, or combinations of these $\dagger$. If only two conformations are assumed to be present then from relation (1) one obtains:

$$
K=\frac{N_{2}}{N_{1}}=\frac{X_{1}-X}{X-X_{2}}
$$

$\dagger$ The use of rate and equilibrium constants in equation (6) was once popular but has understandably fallen into disfavour because the systematic errors are often large and are unpredictable $^{1 \mathrm{~b}}$. 


\section{SUBSTITUENTS LACKING CONICAL SYMMETRY}

(a pair of shiral conformations in place of one of the conformations in (6) introduces only a trivial complication, a factor 2 , which for convenience will be left out in what follows). It is obvious that since $K$ is estimated from a ratio of differences and $X_{1}$ and $X_{2}$ cannot be directly observed, the applications of equation (6) are sensitive to experimental and systematic errors. The effects of such errors will be smallest when $K$ is near 1 and will increase rapidly as $K$ or $K^{-1}$ becomes larger although, paradoxically, they can sometimes be made relatively small for rather large values values of $K$ or $K^{-1}$.

The application of equation (6) to determining the equilibria within the axial and equatorial sets of conformations of 5-isopropyl-1,3-dioxane (Figure 2) necessitated measuring the coupling constants $J_{H_{H}}$ in cis- and trans- 2-methyl-5-isopropyl-1,3-dioxane, which provided models for the axial and equatorial sets of conformations of 5-isopropyl-1,3-dioxane IV itself (Figure 2), and estimating the gauche and trans coupling constants for the individual conformations $\left(X_{2} \text { and } X_{1}\right)^{15}$. The chemical shifts of the protons in $\mathrm{V}$ and $\mathrm{VI}$ are exceptionally favourable owing to the selective deshielding of axial substituents at $\mathrm{C}-5$ by the oxygen atoms and the first order analysis used should be adequate. The individual coupling constants were estimated from a Karplus type equation:

$$
J_{\theta}=A+B \cos \theta+C \cos 2 \theta
$$

for which $A$ was obtained from Abraham and Pachler's empirical relationship for the effect of electronegativity on vicinal coupling constants ${ }^{24}$, $J_{60^{\circ}}$ was taken from adamantane ${ }^{25}$ and $J_{0^{\circ}}-J_{180^{\circ}}$ was taken to be $-2 \mathrm{~Hz}^{15}$, to give values of $B$ and $C$. Because the equilibrium constants are quite near 1 the uncertainties resulting from the use of (7) are small for the mole fractions and very small for the entropies of mixing, which agree quite well with the experimental $\Delta S_{e \rightarrow a}$. A more accurate result could be obtained if a good model for $J_{180^{\circ}}$ could be found. Most models for trans vicinal coupling constants in acyclic systems have been very crude. For example, the mutual repulsion of the $t$-butyl groups in di-t-butylacetaldehyde VIII ${ }^{26}$ (Figure 3 ) must distort the bond angles substantially from those found in isobutyraldehyde VII so that VIII cannot be expected to provide a reliable value of $J_{180^{\circ}}$ for aldehydes. In the adamantane derivative IX, however, the bond angles are normal but the equilibrium is strongly biased and leads to a substantially higher $J_{180^{\circ}}$ than does VIII. Whereas tetramethyladamantane derivatives are suitable for determining $J_{180^{\circ}}$ for fragments of the type $\mathrm{H}-\mathrm{C}\left(\mathrm{sp}^{2}\right)-\mathrm{C}\left(\mathrm{sp}^{3}\right)-\mathrm{H}$, simple adamantane derivatives are suitable for saturated systems $\mathrm{H}-\mathrm{C}\left(\mathrm{sp}^{3}\right)-\mathrm{C}\left(\mathrm{sp}^{3}\right)-\mathrm{H}$. The adamantane $X^{27}$ leads to a value of $J_{180^{\circ}}$ for acetals which is rather higher than that found by curve fitting for XI but which agrees well with a coupling constant obtained from axial protons in a 2 -alkoxytetrahydropyrane ${ }^{28}$. The synthesis of the adamantane derivatives XII and XIII required as models for $J_{180^{\circ}}$ in cis- and trans-2-methyl-5isopropyl-1,3-dioxane $\mathrm{V}$ and VI have yet to be completed.

Dipole moments may be used in equation (6) in much the same way as coupling constants to determine equilibria within axial and equatorial sets of conformations, for example, in 5-methoxy-1,3-dioxane ${ }^{29}$, and, combined 
<smiles>CC(C)C=O</smiles><smiles>C#CC</smiles>

VII

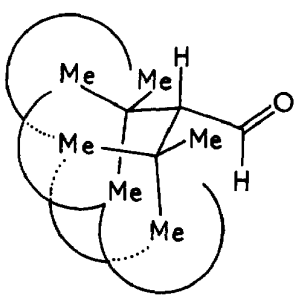

VIII<smiles>CC1(C)COC(C2C3CC4CC(C3)CC2C4)OC1</smiles>

$\mathrm{X}$<smiles>CC1OCCCOC(C)C1C1CC2CC3CC(C2)CC1C3</smiles>

XII<smiles>CC(C)C=O</smiles><smiles>CC12CC3(C)CC(C)(C1)C(C)(C)C(C=O)(C2)C3</smiles>

IX<smiles>CCC1(C)COC(C(C)C)OC1</smiles>

XI

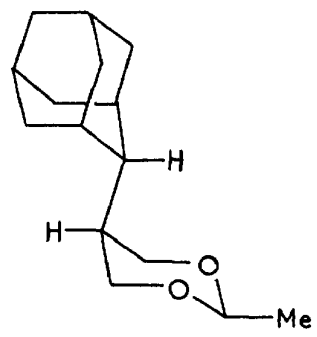

XIII

Figure 3. The conformational equilibrium in isobutyraldehyde and model compounds for estimating $J_{180^{\circ}}$. The distortion to be expected for the acyclic aldehyde VIII makes this a poor model for isobutyraldehyde (VII), but the bond angles in the adamantane IX are close to the normal values. 


\section{SUBSTITUENTS LACKING CONICAL SYMMETRY}

with coupling constants, in 2-alkoxytetrahydropyranes ${ }^{30}$. Dipole moments have the advantage that approximate values for individual conformations can be predicted for almost any structure so that qualitative and semiquantitative estimates of conformational equilibrium constants can be made for many polar molecules. Two difficulties, however, are sometimes overlooked. Dipoles are not in general directed along the axes of polar bonds, unless the symmetry of the molecule requires them to be, and the angles found by microwave spectroscopy (up to $14^{\circ}$ ) are far from negligiblet.

A second difficulty is that group moments are often identified with the moments of simple molecules. For example, the moments of 2-alkoxytetrahydropyranes ${ }^{30}$ and 5-methoxy-1,3-dioxanes ${ }^{29}$ have been estimated from the moments of the parent heterocyclic molecules and simple ethers. The difference of moment between tetrahydropyrane and diethyl ether probably results from the difference in shapes, the former having only gauche arrangements about the skeletal bonds while the trans conformation is strongly favoured in the latter ${ }^{31}$. In some of the conformations of 5-methoxy-1,3dioxanes, however, there are gauche arrangements for the alicyclic ether moieties, and ordinary ethers are therefore imperfect models. This source of error does not appear to have been considered before.

Nuclear magnetic resonance chemical shifts and coupling constants have been widely used in conformational analysis. Proton-proton coupling constants have the advantage that they are relatively insensitive to temperature and solvent effects and where a comparison can be made between estimates of free energies derived from integration at low temperatures ${ }^{14,32}$ and from vicinal coupling constants ${ }^{15}$ the results agree, as mentioned earlier. Unfortunately the differences in coupling constants between conformations is not large and for the relatively complicated spin systems present in many six-membered ring compounds coupling constants cannot always be measured with high precision. This difficulty may be diminished by specific deuterium labelling, preferably with deuterium decoupling, but the problems of synthesis may then be considerable. Chemical shifts, however, are much more sensitive to conformational changes than are coupling constants, but are also much more sensitive to solvent and temperature. The precision obtainable is illustrated by the detection of an isotope effect on a conformational equilibrium ${ }^{16}$. The 1,3-dioxane XIV (Figure 4) was chosen because $(a)$ axial 2-alkyl groups in 1,3-dioxanes experience severe repulsions from syn-axial hydrogen atoms ${ }^{17-19,32},(b)$ the two 2 -substituents lead to a nearly balanced equilibrium, which is necessary if small changes of free energy differences are to be detected, and $(c)$ the chemical shifts of the geminal methyl groups are well separated $(\delta \simeq 0.4$ p.p.m.) and if the difference is used, rather than the individual chemical shifts, the two conformations differ by $2 \delta \simeq 0.8$ p.p.m. The observed isotope effect is $17 \mathrm{cal} / \mathrm{mole}$, i.e. about 0.4

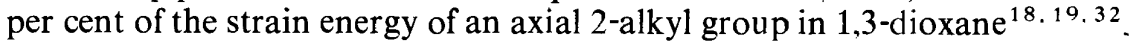

$\dagger$ Since an error of $10^{\circ}$ in the angle between the dipoles in one of the conformations of an $N$-alkylpiperidine could change the derived equilibrium constants by as much as a factor of 2 , which would remove much of the discrepancy with the results from kinetically controlled protonation mentioned earlier, it is important that the assumed values could be checked independently ${ }^{11,29}$, but not all workers in this field have been so careful. 
<smiles>[Y6]C1C2CC1C([Y6])(S)C(C)(C([R])([R])[X])O2</smiles>

XIVa: $\mathrm{R}=\mathrm{CO}_{2} \mathrm{Et}$<smiles>[X][R4]C1(C[R])OC2CCC(O2)C1([Y16])[Y6]</smiles>

$\mathrm{XIVb}: \mathrm{R}=\mathrm{CO}_{2} \mathrm{Et}$

$$
\begin{aligned}
& \mathrm{R}=\mathrm{H}: K_{310(238)}=0.7008(0.6184) \\
& \mathrm{R}=\mathrm{D}: K_{310(238)}=0.6826(0.5953) \\
& K_{\mathrm{H}} / K_{\mathrm{D}}=1.02_{7}\left(1.03_{9}\right) \\
& G=-16_{.0}(-18.0) \mathrm{cal} / \mathrm{mole} \\
& \text { ( } \mathrm{X}=\mathrm{CH}_{3} \mathrm{CH}_{2} \mathrm{OH}, \mathrm{CH}_{2} \mathrm{OAc} \text {, and } \mathrm{CH}_{2} \mathrm{OTos}, K>1 \text { ) }
\end{aligned}
$$

Figure 4. Deuterium isotope effest on the conformational equilibrium in 2,5,5-trimethyl-2ethoxycarbonylmethyl-1,3-dioxane ${ }^{16}$. In a control experiment it was shown that there was no isotope effect (to within $0.1 \%$ ) on $\delta$ in a conformationally biased compound, 2,5,5-trimethyl-2$t$-butyl-1,3-dioxane, used to estimate $\delta$ for XIVa and XIVb.
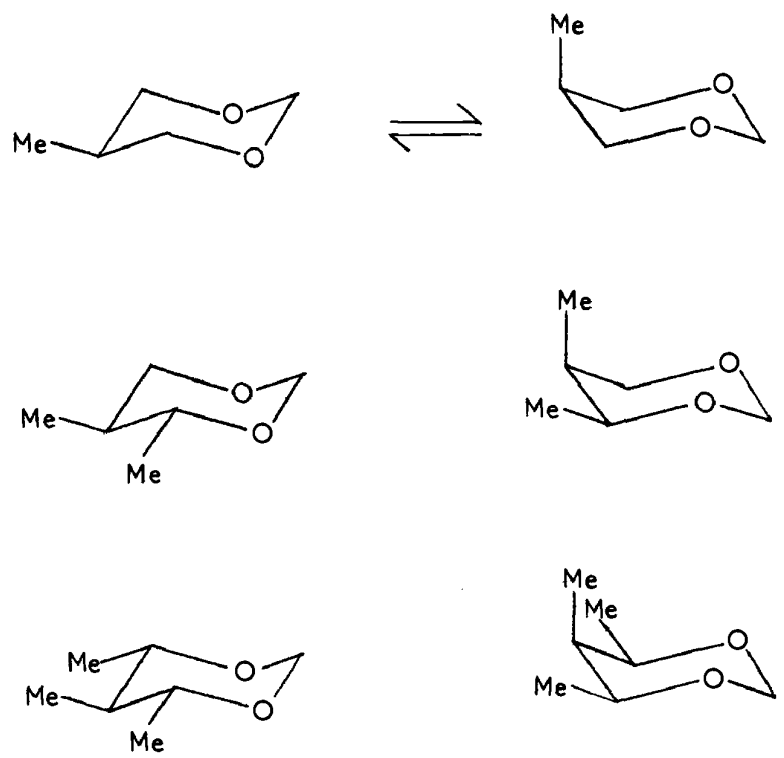

Figure 5. The conformations of 5-methyl-1,3-dioxane and the two series of model compounds used to estimate the temperature dependence of the difference between the chemical shifts of the C-2 geminal protons. 


\section{SUBSTITUENTS LACKING CONICAL SYMMETRY}

and is substantially independent of temperature. If it is accepted that owing to smaller amplitudes of vibration deuterium is smaller than hydrogen then $K$ must be $<1$ for XIV but $>1$ (as expected) for other groups $X=\mathrm{CH}_{3}, \mathrm{CH}_{2} \mathrm{OH}, \mathrm{CH}_{2} \mathrm{OAc}$. This is a result which would not be easily obtained by other methods of studying conformational equilibria, and may probably be explained by dipole-dipole repulsions but the latter are difficult to estımate for ester groups. Only chemical shifts provide sufficient sensitivity for the reliable detection of such small changes of conformational equilibria.

In the previous example systematic errors in estimating equilibrium constants from chemical shifts of mobile and locked compounds largely cancel and the isotope effect, $\Delta \Delta G$, is much more accurate than the individual values of $\Delta G$. The estimation of values of $\Delta G_{e \rightarrow a}$ using chemical shifts derived from either model compounds or from conformations at low temperatures has been strongly criticised ${ }^{32}$. It has been suggested that model compounds should be used to provide corrections for the temperature dependence of the chemical shifts of conformations directly observed at low temperatures, and in this way results based on using chemical shifts in equation (6) may be made more reliable. Unfortunately, model compounds cannot be relied on even to estimate the temperature dependence of chemical shifts of conformations. This has been demonstrated for 5-methyl-1,3-dioxane by using two series of model compounds (Figure 5), for which the temperature effects are different. Even when the two series of model compounds are used to estimate the temperature dependence of the chemical shifts differences for the geminal C-2 protons of the individual conformations by extrapolation the results are not very good. Although the free energy differences $\Delta G_{e \rightarrow a}$ agree reasonably well with the values obtained by integration at low temperatures and from vicinal coupling constants, for the solvents $\mathrm{Me}_{4} \mathrm{Si}, \mathrm{CCl}_{3} \mathrm{~F}$, and $\left(\mathrm{CD}_{3}\right)_{2} \mathrm{CO}$, but not for $\mathrm{CD}_{3} \mathrm{OD} \dagger$, the entropy differences $\Delta S_{e \rightarrow a} \simeq-1$ e.u. estimated for a relatively small temperature interval, are clearly not satisfactory ${ }^{16,32}$. Since solvent effects are very marked in 1,3-dioxanes it may well be that chemical shifts can be used more reliably in other systems but great care must be exercised.

When equilibria are strongly biased with $K \gg 1$ equation (6) can only be used for chemical shifts if $X_{2}(\simeq X)$ can be estimated with precision, although only very rough estimates of $X_{1}$ may be needed. This can sometimes be achieved if the chemical shifts $X$ can be measured over a wide temperature range, say $200^{\circ}$, from low temperatures at which it is almost identical with $X_{2}$ so that the validity of a model compound for estimating the temperature dependence of $X_{2}$ can be verified to higher temperatures at which $X$ and $X_{2}$ differ by an easily measurable amount. This is apparently successful for the sulphite of neopentylene glycol, using 2-t-butyl-5,5-dimethyl-1,3-dioxane as a model for the chemical shifts of the geminal methyl groups, and gives $\Delta G_{e \rightarrow a}=2.5 \pm 0.4 \mathrm{kcal} / \mathrm{mole}^{33}$, i.e. the conformation with the exocyclic oxygen atom axial is strongly favoured ${ }^{34}$. When this method is extended to even more biased equilibria, e.g. as in 2,5,5-trimethyl-1,3-dioxane $\left(\Delta G_{e \rightarrow a} \simeq+4 \mathrm{kcal} / \mathrm{mole}^{18,19}\right)$, for which the maximum value of $X-X_{2}$ was only 0.007 p.p.m., at $+180^{\circ}$, the results depend very critically upon the

$\dagger$ The results are far from complete for this solvent and the discrepancy may not be real. 


\section{J. T. ROBINSON}

validity of the model compound. Even in this extreme example the derived value $\Delta G_{e \rightarrow a} \simeq+3.5 \mathrm{kcal} / \mathrm{mole}$ is in fair agreement with other estimates.

When two or more polar groups are present in a molecule, whether as substituents or as part of a ring, electric dipole-dipole interactions become significant in conformational and stereochemical equilibria. Examples are to be found in 1,3-diazanes and 1,3,5-triazanes (the Rabbit-ear Effect, vide infra), where the polar groups are part of the ring, in 2-substituted tetrahydropyrans and sugar derivatives generally (the Anomeric Effect) ${ }^{1 \mathrm{c}}$, where one polar group is part of the ring, and in di- or polysubstituted cyclohexanes. Owing to the complexity resulting from rotating dipole groups, most of the more carefully studied systems until recently have been concerned with simple symmetrical polar substituents.

Most attempts to correlate dipole-dipole interactions with their effect on conformational equilibria have made use of the point dipole approximation, i.e. the dipole resulting from the difference of position of the centroids of negative and positive charge throughout a molecule, or some part of it (a polar bond or group) is approximated by a vanishingly short dipole with the same magnitude situated at some point along a polar bond and directed along the axis of that bond. Although it is quite easy to carry out calculations of electrostatic interactions with more complicated and less naive assumptions the simplest point dipole calculations are not without value, and even if nothing better is attempted they should always be carried out before attributing differences in conformational equilibria to dipole-dipole interactions $\nmid$. The choice of position of point dipoles has been quite wide, e.g. from $0.77 \AA$ (the covalent radius of carbon) to $1.70 \AA$ (seven-eighths of the $\mathrm{C}-\mathrm{Br}$ bond length) from carbon along the $\mathrm{C}-\mathrm{Br}$ bond in 2-bromocyclohexanone. It is worth mentioning that replacing point dipoles situated at the middle of bonds by equal moments represented by appropriate fractional charges on the atoms greatly simplifies the computations but often has only marginal effects on the calculated energies ${ }^{33}$ and seems more realistic physically. The interaction energy $E \mu$ between two point dipoles $\mu_{1}$ and $\mu_{2}$ at a distance $r$ apart is given by ${ }^{36}$ :

$$
E \mu=\frac{\mu_{1} \mu_{2}}{r^{3} D}\left(\cos X-3 \cos \alpha_{1} \cos \alpha_{2}\right)
$$

$D$ is the dielectric constant, $X$ is the angle between the dipoles, and $\alpha_{1}$ and $\alpha_{2}$ are the angles between each of the dipoles and the line joining them. It is desirable to use $\alpha_{2}$ and not its complement, as was pointed out by Lehn and Ourisson $^{35}$ ! Even when there is no direct diffraction evidence for the shape and dimensions of a six-membered ring the geometrical parameters $r, X, \alpha_{1}$, and $\alpha_{2}$ can usually be calculated with fair accuracy from standard bond lengths and angles, or, much less satisfactorily, measured from models. The choice of dihedral angles for gauche conformations is less easily made but in a number of instances the computed energies $E \mu$ are not very sensitive to $10^{\circ}$ changes in

$\dagger$ The converse is also true: dipole-dipole interactions should not be dismissed as an important factor on the basis of intuitive qualitative arguments, as was done by Wood and Woo ${ }^{35}$ when discussing the conformational equilibria in trans-1,4-dihalocyclohexanes. 
dihedral angles ${ }^{33}$. The dipole moments $\mu_{1}$ and $\mu_{2}$ may be more troublesome to estimate because if two strongly polar groups are close together their mutual polarization changes the resultant molecular moment from the value predicted on the basis of simple vectorial addition of moments obtained from molecules with only one polar group. One solution is to ignore mutual polarization, as seems to be common in studies of the anomeric effect ${ }^{30,37}$. A better but not always easily applied approximation is to reduce $\mu_{1}$ and $\mu_{2}$ to values whose vector sums equal the moments of model compounds of fixed conformation ${ }^{38}$. When the dipoles are not close together their mutual polarization is small and can either be calculated by accepted approximate methods $^{39}$, or neglected as being unimportant in comparison with other sources of error. Finally there is the dielectric constant to be considered. This is not taken to be the dielectric constant of the solvent, but is an 'internal' dielectric constant supposed to represent the polarizability of the molecule between and around the point dipoles. Anderson and Sepp ${ }^{37}$ have argued in favour of different values of $D$ (from 1.0 to 2.4) for different situations, but more commonly 2 has been adopted, following Kirkwood and Westheimer ${ }^{40}$, for reasons which have been most clearly set out by the Allingers ${ }^{38}$. Unfortunately the arguments in favour of using a dielectric constant $>1$ inside a molecule in these applications of equation (8) are nonsense. When the real dipole moment of a molecule is replaced by an equivalent point dipole the latter must be placed somewhere in a hypothetical molecule which is not polarizable so far as that dipole is concerned, since a molecule is not polarized by its own dipole!

It is not surprising, therefore, that when two dipoles are quite well separated, as in 4-hydroxycyclohexanones ${ }^{41}, 3 \alpha$-substituted $5 \alpha$ - and $5 \beta$ cholestan-6-ones $^{42}$, trans-1,4-dichlorocyclohexane ${ }^{35}$, and 4-chlorocyclohexanone ${ }^{43}$ the differences between the observed free energy differences for conformational or stereochemical equilibria and estimates of steric strain energies can usually be accounted for dipole-dipole interactions provided the dielectric constant is assumed to be unity ${ }^{33}+$. Such calculations tend to overestimate dipole-dipole interactions because the effect of the solvent is neglected but this is no reason for using a dielectric constant $>1$ within the molecule. When the calculated dipole-dipole interactions are too small to account for the observed effect of a polar substituent, as seems to be the case with, e.g. 3-methoxycyclohexanone ${ }^{33,44}$, it is legitimate and desirable to consider what other influence may be operating.

In the examples so far the location of the point dipoles has not been critical and my purpose has been to show that an internal dielectric constant of unity is appropriate. It has been recognized for forty years, however, that bond moments derived from small molecules with a single polar group, in the ordinary sense, cannot always be used successfully to predict the moments of more complex molecules because the dipolar groups in the latter polarize one another, as mentioned earlier. and induce dipoles in polarizable but

\footnotetext{
$\dagger$ Even if the appropriate locations of the point dipoles could be detected independently, there would still be the mutual polarization of the dipolar groups and solvent effects to be accounted for before good agreement between calculated and 'observed' electrostatic interactions could be general, assuming that the point dipole model is adequate.
} 
nominally non-polar parts of a moleculet. The effect of the induced moments is to influence not only the magnitude of the moment and frequently its direction but also the location of the equivalent point dipole. The carbonyl compounds in Figure 6 illustrate the point. The moment of cyclohexanone is not significantly different from that of acetone, showing that induced moments in the three methylene groups not directly attached to the carbonyl group are small and I will ignore them in what follows.

The magnitude and directions of the moments of acetaldehyde and acetone are reproduced quite well if the carbonyl group is assigned a constant moment of $2.34 \mathrm{D}$ as in formaldehyde and induces a moment of $0.56 \mathrm{D}$ along each $\mathrm{C}-\mathrm{Me}$ bond relative to $\mathrm{C}-\mathrm{H}$. It is clear that the induced moments move the equivalent point dipole for the molecular moment away from the oxygen atom along the axis of the $\mathrm{C}=\mathrm{O}$ as one goes from formaldehyde to cyclohexanone in Figure 6. Thus if the equivalent point dipole for a polar bond should be placed half-way along that bond, which appears to be the simplest as well as the commonest assumption, the equivalent point dipole for a polar molecule or part of a molecule should not be placed at the middle of the polar bond but rather nearer to the middle of the molecule + . Thus if the substituents are more or less on opposite sides of the molecule calculations using a point dipole located at the centre of each of the polar bonds will underestimate dipole-dipole interactions by overestimating the distance between the dipoles. Trans-1,4-dichlorocyclohexane ${ }^{33,35}$ illustrates the sort of difference to be expected (Table 2) and this may explain why Stolow and Groom ${ }^{40}$ found that the calculated dipole-dipole interactions in 4-hydroxycyclohexanone are a little too small to account for the observed effect of the carbonyl group on the conformational energy of the hydroxyl group,

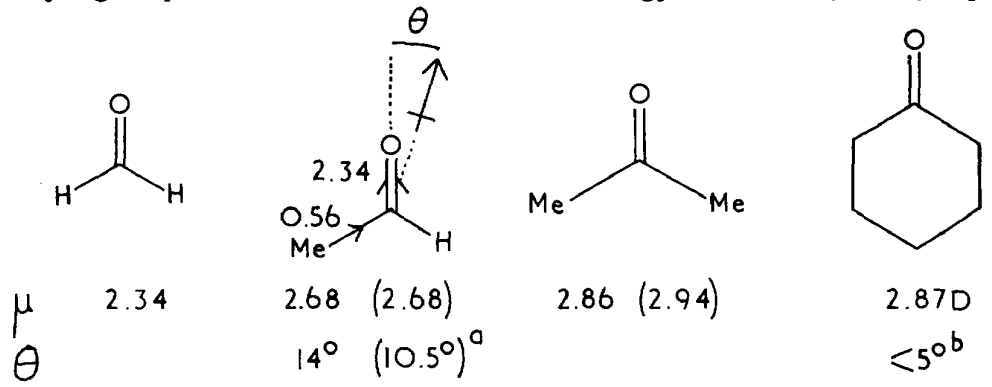

Figure 6. Dipole moments of some simple carbonyl compounds derived from microwave spectroscopy. The angles $\theta$ are the angles between the moment and the axis of the carbonyl group. The values in brackets are calculated using the most naive assumptions about the polarization of a methyl (or methylene group) immediately adjacent to the carbonyl group, as implied by the bond moments shown on the formula of acetaldehyde. $\left({ }^{\mathrm{a}}\right.$ In the nodal plane of the carbonyl double bond: ${ }^{b}$ perpendicular to the nodal plane of the carbonyl group; the small value is consistent with the relative unimportance of polarization of the 3-, 4-, and 5-methylene groups in cyclohexanone suggested by the similarity of the dipole moments of acetone and cyclohexanone, although this may result from the near cancellation of opposing effects.)

$\dagger$ Rather complex but essentially empirical schemes for estimating induced moments have been developed but the simple description given here is more suited to bring out the points I wish to make.

$\ddagger$ In practice it is more convenient to treat the total moment as made up of two or more partial moments placed at the midpoints of bonds rather than find the location appropriate to a single point dipole. 
although uncertainties about the conformations of axial cyclohexanols ${ }^{12,13}$ make such calculations more uncertain than usual.

When the dipoles are closer together than the previous examples the location of the point dipoles may be more critical. Thus in 1,3,5-triazanes the calculated dipole-dipole interactions favouring conformations with one $N$-substituent axial, the Rabbit-ear Effect ${ }^{45}$, are $1.5 \pm 0.2 \mathrm{kcal} / \mathrm{mole}$ if the point dipoles are located at the nitrogen atoms, being considered to be mainly due to the unshared pairs, or $1.0 \pm 0.15 \mathrm{kcal} / \mathrm{mole}$ if the dipoles are located at the midpoints of the $\mathrm{C}-\mathrm{N}$ bonds. Unfortunately, the steric interactions cannot be estimated with any certainty so that a choice between the two locations cannot be made and it may be that independent evaluation of the latter may lead to a proper estimate of the steric interactions.

I turn finally to molecules where the dipoles are close together, as in cyclohexanones with polar substituents at $\mathrm{C}-2$. It is undoubtedly true that the usual point dipole calculations seriously overestimate the apparent dipoledipole interactions in 2-chloro- and 2-bromo-cyclohexanone unless the dielectric constant is given the nonsensical value of 2 or more. The situation is even worse in 2-fluorocyclohexanone, and even if the moments induced in adjacent methylene groups by the polar $\mathrm{CO}$ and $\mathrm{CHal}$ bonds are taken into account (this lowers the dipole-dipole interactions, in contrast to some earlier examples), simple steric and electrostatic effects do not account for the observed conformational equilibria and the discrepancy is greatest for fluorine. The equilibria in 2-methoxy- and 2-acetoxy-cyclohexanone are perhaps even more surprising, because both steric and electrostatic interactions probably favour the axial conformation in the former, yet the equatorial conformations are more stable by $1.0^{46}$ and $\varangle 2^{47} \mathrm{kcal} / \mathrm{mole}$. House and Frank ${ }^{46}$ considered $1.0 \mathrm{kcal} / \mathrm{mole}$ to agree well with the value, $\Delta G_{e \rightarrow a}=0.6$ to $0.7 \mathrm{kcal} / \mathrm{mole}^{1 \mathrm{c}}$, found for methoxycyclohexane, but this comparison is a good example of mistakenly transferring conformational energies of a substituent from one molecule to another providing a very different environment. The comparison overlooks the dipole-dipole interactions favouring an axial methoxy group ${ }^{33}$, as well as the important differences between gauche interactions between $\mathrm{O}-\mathrm{Me}$ and $\mathrm{CH}_{2}(=1.5$ $\mathrm{kcal} / \mathrm{mole}^{31}$ ), which are unavoidable in methoxycyclohexane and in equatorial 2-methoxy-cyclohexanone, and between $\mathrm{O}-\mathrm{Me}$ and the $\pi$ electrons of the $\mathrm{CO}$ (which is likely to be very small) when the methoxy group is axial in the ketone, cf. 2-ethylcyclohexanone ${ }^{21}$. Similar arguments apply to the 2-acetoxy ketone but the calculation of steric and dipole-dipole interaction is less certain for esters ${ }^{33}$. It would appear that steric and electrostatic effects are not sufficient to account for conformational equilibria in 2 -substituted cyclohexanones. It has been suggested ${ }^{48}$ that an electronic effect with the steric requirements of hyperconjugation (or ' $\alpha$-hydrogen bonding' $)^{49}$ is required to account for the conformational properties of carbonyl compounds, with a value of $1 \mathrm{kcal} / \mathrm{mole}$ for each suitably oriented $\alpha-\mathrm{CH}$ bond. The results now available suggest that the effect is even greater when a first period element with unshared pairs of electrons is directly attached to the $\alpha$-carbon atom. Hyperconjugation, as an energetically significant effect on ground states of molecules, became unfashionable after Dewar's demolition ${ }^{50}$ of earlier evidence supposed to demonstrate it but it is 


\section{J. T. ROBINSON}

Table 2. Some examples ${ }^{a}$ of the magnitude of dipole-dipole interactions in some six-membered ring compounds with relatively well separated polar groups<smiles>O=C1CCC(Cl)CC1</smiles>

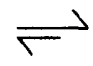<smiles>ClC1CCCC(Cl)CC1</smiles>
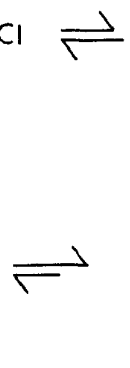<smiles>CO[C@H]1CCCC(=O)C1</smiles>
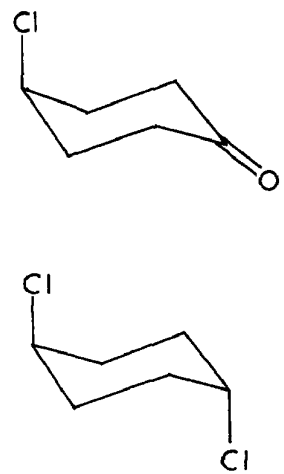

XVI

XVII

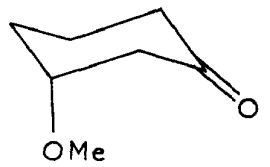

$\mathrm{OMe}$<smiles>C/C=C\CN(C)CN(C)C</smiles>

XVIII

Energies $(\text { kcal/mole })^{b}$

Compound

$\Delta G_{e \rightarrow a}^{c} \quad$ Steric ${ }^{d} \quad$ Difference $\quad \begin{gathered}\Delta E \mu^{f} \\ (\text { calculated })\end{gathered}$

\begin{tabular}{lcccc} 
XV & -0.4 & +0.4 & -0.8 & -1.1 \\
& $\left(\mathrm{CCl}_{4}\right)$ & & & -1.4 \\
XVI & +0.1 & +0.8 & -0.7 & $(-1.1)^{g}$ \\
& $\left(\mathrm{C}_{2} \mathrm{D}_{2} \mathrm{Cl}_{4}\right)$ & & & -0.6 \\
XVII & -0.4 & +0.4 & -0.8 & $-1.0^{h}$ \\
XVIII & $\left(\mathrm{CH}_{3} \mathrm{OH}\right)$ & - & - & $(-1.5)^{i}$ \\
\hline
\end{tabular}

a Other examples may be found in references 41 and 42 .

b A positive sign implies that the conformation with equatorial groups is preferred.

c Observed (solvent in brackets).

- Calculated from an appropriate compound with only one polar group.

e Observed free energy difference minus the steric energy.

$f \mathrm{Calculated}$ using fractional charges on atoms, with an allowance being made for the polarization of methylene groups adjacent to the polar groups, using a dielectric constant of unity.

* Calculated using fractional charges on chlorine and $\alpha$-carbon atoms only, to illustrate the sort of erroes which may be expected from treating polar groups as single dipoles located in one bond.

${ }^{h}$ For one $N$-methyl group going from equatorial to axial. No reliable estimates of the observed free energy differences or steric energies are available for diazanes, but the effect is qualitatively obvious 29,45 .

' Calculated for point dipoles located at the nitrogen atoms, to illustrate the importance of correctly locating dipoles or else considering alternative locations. 


\section{SUBSTITUENTS LACKING CONICAL SYMMETRY}

now once more respectable. In particular, calculations which lead to rather good estimates of dipole moments of simple molecules, a sensitive test, indicate an alternation of charge even in saturated compounds as a result of donation of $\pi$-electrons by nitrogen, oxygen, or fluorine to $\alpha$-hydrogen atoms, as well as the expected withdrawal of $\pi$-electrons by carbonyl ${ }^{51}$. It is obvious that these two effects will be cooperative to the greatest extent in an $\alpha$ substituted carbonyl compound when the $\alpha$-substituent is in the nodal plain of the $\pi$-electrons of the carbonyl group.

In conclusion I would like to make the following points about the conformational analysis of six-membered rings with substituents of low symmetry:

a. Methods for determining equilibria within axial and equatorial sets of conformations need further development, and new methods need to be introduced. As an example of the latter I single out neutron scattering

b. More information is required about the relative directions of dipole moments and polar bonds, particularly for unsymmetrical substituents†.

c. When two or more polar groups are present the importance of electrostatic interactions should be estimated before even qualitative interpretations of conformational equilibria are attempted.

d. Model compounds used for estimating the properties of conformations should be chosen with great care and if possible two or more sets should be used to provide cross-checks.

I must thank Professor J. S. Anderson for generously making available the $100 \mathrm{MHz}$ nuclear magnetic resonance spectrometer in the Inorganic Chemistry Laboratory in Oxford for variable-temperature measurements, and my present and former research students Drs B. J. Armitage, W. D. Cotterill, N. W. J. Pumphrey and F. G. Riddell, and Messrs F. C. Ball, A. P. Foster, P. J. Knowles, J. D. Schofield, H. J. F. Tarratt and M. G. Ward.

\section{REFERENCES}

${ }^{1}$ E. L. Eliel, N. L. Allinger, S. J. Angyal and G. A. Morrison. Conformational Analysis, Interscience; New York (1965), (a) p 1. (b) pp 71 et seq., (c) pp 411 et seq., (d) p 438.

2 F. Bell and J. Kenyon. Chem. \& Ind, 4. 864 (1926);

W. H. Mills, Chem. \& Ind. 4, 884 (1926).

3 C. C. Costain and J. M. Dowling. J. Chem. Phys. 32. 158 (1960).

${ }^{4}$ S. I. Chan, J. Zinn, J. Fernandez and W. G. Swinn. J. Chem. Phys. 33, 1643 (1960).

5 S. I. Miller. Advances in Physical Organic Chemistry, Vol. 6, (V. Gold, Ed.), Academic Press: New York (1968).

6 J. B. Hendrickson. J. Amer. Chem. Soc. 83, 4537 (1961).

7 B. J. Armitage, G. W. Kenner and M. J. T. Robinson. Tetrahedron, 20, 723, 747 (1964).

8 A. Booth. Chem. Commun. 802 (1968).

9 J. McKenna and J. M. McKenna. J. Chem. Soc. (B). 644 (1969).

10 M. G. Ward, B.A. (Chemistry), Part II, Thesis, Oxford (1969).

${ }^{11}$ R. J. Bishop, L. E. Sutton, D. Dineen, R. A. Y. Jones, A. R. Katritzky and R. J. Wyatt. J. Chem. Soc. (B), 493 (1967).

$\dagger$ Mr J. D. Schofield has begun work which should lead to such a measurement for aryl-C bonds in arylcyclohexanes. Dr N. W. J. Pumphrey in a joint project with Dr L. E. Sutton and his collaborators Dr R. J. Bishop and Mr C. W. Atkin in the Physical Chemistry Laboratory, Oxford, has obtained a value of $1 \pm 1^{\circ}$ for cyanocyclohexane. Substituents which are not axially symmetrical, however, present great difficulties. 


\section{J. T. ROBINSON}

12 L. Joris, P. von R. Schleyer and E. Osawa. Tetrahedron, 24, 4759 (1968).

13 R. D. Stolow and A. A. Gallo. Tetrahedron Letters, 3331 (1968).

14 F. R. Jensen, C. H. Bushweller and B. H. Beck. J. Amer. Chem. Soc. 91,344 (1969).

15 P. J. Knowles, B.A. (Chemistry), Part II, Thesis, Oxford (1968).

16 F. C. Ball, B.A. (Chemistry), Part II, Thesis, Oxford (1969).

17 F. G. Riddell and M. J. T. Robinson. Tetrahedron, 23, 3417 (1967).

18 E. L. Eliel and Sr. M. C. Knoeber. J. Amer. Chem. Soc. 90, 3444 (1968).

19 K. Pihlaja and S. Luoma. Acta Chem. Scand. 22, 2401 (1968);

K. Pihlaja and Heikkila. Acta Chem. Scand. 21, 2390; 2430 (1967).

20 E. L. Eliel and M. C. Reece. J. Amer. Chem. Soc. 90, 1560 (1968).

21 W. D. Cotterill and M. J. T. Robinson. Tetrahedron, 20, $765 ; 777$ (1964).

22. K. M. Wellman, W. S. Briggs and C. Djerassi. J. Amer. Chem. Soc. 87, 73 (1965).

${ }^{23}$ M. J. T. Robinson. Chem. \& Ind. 932 (1964).

${ }^{24}$ R. J. Abraham and K. G. R. Pachler. Molec. Phys. 7, 165 (1963).

25 R. C. Fort and P. von R. Schleyer. J. Org. Chem. 30, 789 (1965).

26 G. J. Karabatsos and N. Hsi. J. Amer. Chem. Soc. 87, 2864 (1965).

27 H. J. F. Tarratt, D.Phil. Thesis, Oxford (1969).

28 F. G. Riddell, Ph.D. Thesis, Liverpool (1965)

29 A. R. Katritzky, personal communication.

30 A. J. de Hoog, H. R. Busy, C. Altona and E. Havinga. Tetrahedron, 25, 3365 (1969).

31 T. Kitagawa and T. Mihazawa. Bull. Chem. Soc. Japan, 41, 1976 (1968).

${ }^{32}$ F. R. Jensen and B. H. Beck. J. Amer. Chem. Soc. 90, 3251 (1968); F. A. L. Anet and P. M. Henrichs. Tetrahedron Letters, 741 (1969).

${ }^{33}$ M. J. T. Robinson, unpublished results and calculations.

34 H. F. van Woerden and E. Havinga. Rec. Trav. Chim. Pays-Bas 86, 353 (1967).

35 G. Wood and E. P. Woo. Canad. J. Chem. 45, 2477 (1967).

36 J.-M. Lehn and G. Ourisson. Bull. Soc. Chim. France, 1113 (1963).

${ }^{37}$ C. B. Anderson and Sepp, Tetrahedron, 24, 1707 (1968).

${ }^{38}$ N. L. Allinger, J. Allinger, L. A. Freiberg, R. F. Czaja and N. A. LeBel. J. Amer. Chem. Soc. 82, $5876(1960)$, and references therein.

39 E.g. H. M. Smallwood and K. F. Herzfeld. J. Amer. Chem. Soc. 52, 1919 (1930); R. P. Smith and E. M. Mortensen. J. Amer. Chem. Soc. 78, 3932 (1956l.

40 J. G. Kirkwood and F. H. Westheimer. J. Chem. Phys. 6, 506; 513 (1938).

41 R. D. Stolow and T. Groom. Tetrahedron Letters, 4069 (1968).

42 R. Grayshan, Ph.D. Thesis, Sheffield (1968).

${ }^{43}$ D. N. Kirk. Tetrahedon Letters, 1727 (1969).

$44 \mathrm{G}$. H. Whitham, personal communication.

45 R. O. Hutchins, L. D. Kopp and E. L. Eliel. J. Amer. Chem. Soc. 90, 7174 (1968).

${ }^{46}$ H. O. House and G. A. Frank, J. Org. Chem. 30, 2948 (1965).

47 A. P. Foster, B.A. (Chemistry), Part II, Thesis, Oxford (1968).

48 W. D. Cotterill and M. J. T. Robinson. Tetrahedron Letters, 1833 (1963).

49 M. M. Kreevoy and H. Eyring. J. Amer. Chem. Soc. 79, 5121 (1957).

50 M. J. S. Dewar. Hyperconjugation, The Ronald Press' New York (1962).

${ }^{51}$ J. A. Pople and M. Gordon. J. Amer. Chem. Soc. 89, 4253 (1967). 